\title{
Comparative Analysis of Six Lagerstroemia Complete Chloroplast Genomes
}

\section{OPEN ACCESS}

Edited by:

Rongling Wu,

Pennsylvania State University, USA

Reviewed by:

Gaurav Sharma,

University of California, Davis, USA

Jing Wang,

Beijing Forestry University, China

*Correspondence:

Zhili Suo

zlsuo@ibcas.ac.cn

${ }^{\dagger}$ These authors have contributed equally to this work

Specialty section

This article was submitted to Evolutionary and Population Genetics,

a section of the journal

Frontiers in Plant Science

Received: 08 November 2016 Accepted: 04 January 2017 Published: 19 January 2017

Citation:

Xu C, Dong W, Li W, Lu Y, Xie X, Jin X, Shi J, He K and Suo Z (2017) Comparative Analysis of Six Lagerstroemia Complete Chloroplast

Genomes. Front. Plant Sci. 8:15. doi: 10.3389/fpls.2017.00015

\begin{abstract}
Chao $\mathrm{Xu}^{1,2 \dagger}$, Wenpan Dong ${ }^{3 \dagger}$, Wenqing $\mathrm{Li}^{4 \dagger}$, Yizeng $\mathrm{Lu}^{4}$, Xiaoman $\mathrm{Xie}^{4}$, Xiaobai Jin ${ }^{5}$, Jipu Shi ${ }^{6}$, Kaihong $\mathrm{He}^{6}$ and Zhili Suo ${ }^{1 *}$

1 State Key Laboratory of Systematic and Evolutionary Botany, Institute of Botany, Chinese Academy of Sciences, Beijing, China, ${ }^{2}$ University of Chinese Academy of Sciences, Beijing, China, ${ }^{3}$ Peking-Tsinghua Center for Life Sciences, Academy for Advanced Interdisciplinary Studies, Peking University, Beijing, China, ${ }^{4}$ Shandong Provincial Center of Forest Tree Germplasm Resources, Jinan, China, ${ }^{5}$ Beijing Botanical Garden, Institute of Botany, Chinese Academy of Sciences, Beijing, China,

${ }^{6}$ Xishuangbanna Tropical Botanical Garden, Chinese Academy of Sciences, Mengla, China
\end{abstract}

Crape myrtles are economically important ornamental trees of the genus Lagerstroemia L. (Lythraceae), with a distribution from tropical to northern temperate zones. They are positioned phylogenetically to a large subclade of rosids (in the eudicots) which contain more than $25 \%$ of all the angiosperms. They commonly bloom from summer till fall and are of significant value in city landscape and environmental protection. Morphological traits are shared inter-specifically among plants of Lagerstroemia to certain extent and are also influenced by environmental conditions and different developmental stages. Thus, classification of plants in Lagerstroemia at species and cultivar levels is still a challenging task. Chloroplast (cp) genome sequences have been proven to be an informative and valuable source of $\mathrm{cp}$ DNA markers for genetic diversity evaluation. In this study, the complete $\mathrm{cp}$ genomes of three Lagerstroemia species were newly sequenced, and three other published cp genome sequences of Lagerstroemia were retrieved for comparative analyses in order to obtain an upgraded understanding of the application value of genetic information from the $\mathrm{cp}$ genomes. The six cp genomes ranged from 152,049 bp (L. subcostata) to 152,526 bp (L. speciosa) in length. We analyzed nucleotide substitutions, insertions/deletions, and simple sequence repeats in the $\mathrm{cp}$ genomes, and discovered 12 relatively highly variable regions that will potentially provide plastid markers for further taxonomic, phylogenetic, and population genetics studies in Lagerstroemia. The phylogenetic relationships of the Lagerstroemia taxa inferred from the datasets from the $\mathrm{cp}$ genomes obtained high support, indicating that $\mathrm{cp}$ genome data may be useful in resolving relationships in this genus.

Keywords: Lagerstroemia, chloroplast genome, comparative genomics, simple repeat sequence, sequence divergence, plastid marker, phylogeny

\section{INTRODUCTION}

On the earth, some major subclades (i.e., Rosids, Asterids, Saxifragales, Santalales, and Caryophyllales) are recognized phylogenetically under the eudicot clade of angiosperms, consisting of $\sim 75 \%$ of all flowering plant species. Among the subclades, the rosids are grouped together as a large evolutionary monophyletic group, containing more than $25 \%$ of all angiosperms. 
Lagerstroemia plants are positioned phylogenetically in the Lythraceae (within the Myrtales Rchb.) of the rosids among core eudicots. Lagerstroemia, one of the 25 genera in the family Lythraceae, has about 56 species in the world, with a distribution from the tropical to northern temperate zones (Qin and Shirley, 2007; APG III, 2009; Su et al., 2014).

Crape myrtles produce abundant large and beautiful panicles with charming flowers commonly lasting for about 3 months or more across summer and autumn seasons (Qin and Shirley, 2007). Their leaves can clean the air by absorbing smoke and dust. They are well-known excellent ornamental trees for city gardening and environmental protection. Their cultivation has a history of at least 1500 years in China. At present, more than 500 cultivars have been bred in the world. They have significant value in horticultural and landscaping application (Huang et al., 2013a,b,c).

Phylogenetic relationships within Lythraceae have been approached using morphology and DNA evidences from the $r b c \mathrm{~L}$ gene, the $t r n \mathrm{~L}-\mathrm{F}$ region, and the $p s a \mathrm{~A}-y c f 3$ intergenic spacer of the cp genome, and ITS (the internal transcribed spacer) of the nuclear genome (Huang and Shi, 2002; Graham et al., 2005). The four DNA markers ( $r b c \mathrm{~L}, m a t \mathrm{~K}, t r n \mathrm{H}-p s b \mathrm{~A}$, and ITS) can only meet the need for plant identification at/above species level with limited or no resolution among closely related species and/or cultivars (Xiang et al., 2011; Suo et al., 2012, 2015, 2016). Due to shared morphological traits to some extent among species and cultivars, the lack of morphological and DNA markers heavily inhibited the genetic diversity evaluation of Lagerstroemia germplasm resources. Genetic information from comparative genomics for researches on genetic diversity and phylogeny in the Lagerstroemia is limited (Pounders et al., 2007; Wang et al., 2011; Suo et al., 2012, 2015, 2016; He et al., 2014; Gu et al., 2016a,b).

Chloroplasts are key organelles in plants for photosynthesis and other biochemical pathways such as the biosynthesis of starch, fatty acids, pigments, and amino acids (Dong et al., 2013, 2016; Raman and Park, 2016). Chloroplast (cp) genome, as one of the three DNA genomes (the other two are nuclear and mitochondrial genomes) in plant body, with uniparental inheritance, has a highly conserved circular DNA arrangement ranging from 115 to $165 \mathrm{~kb}$. Complete cp genome sequences have been widely accepted as an informative and valuable data source for understanding evolutionary biology because of their relatively stable genome structure, gene content, and gene order (Dong et al., 2012, 2013, 2014, 2016; Suo et al., 2012, 2015, 2016; Curci et al., 2015; Downie and Jansen, 2015; Song et al., 2015). Along with the accumulation of complete $\mathrm{cp}$ genome sequences, comparative study of chloroplast genomes from Lagerstroemia plants is helpful for upgrading our evaluation on the application value of the cp genomes.

In this study, we report three newly sequenced complete $\mathrm{cp}$ genomes from the Lagerstroemia (two species and one cultivar) and genomic comparative analyses with other three published $\mathrm{cp}$ genome sequences of the genus downloaded from the National Center for Biotechnology Information (NCBI) organelle genome database (https://www.ncbi.nlm.nih.gov), focusing on organization, gene content, patterns of nucleotide substitutions, and simple sequence repeats (SSRs). The aims of our study are: (i) to deepen our understanding on the genetic and evolutionary significance from the structural diversity in the cp genomes, (ii) to upgrade our understanding on the application value of the complete cp genomes of Lagerstroemia, and (iii) to provide genetic resources for future research in this genus.

\section{MATERIALS AND METHODS}

\section{Plant Materials and DNA Extraction}

Fresh leaves were collected from the trees of Lagerstroemia subcostata and L. indica "Lüzhao Hongdie" growing in the Beijing Botanical Garden (N 39 $48^{\prime}$, E $116^{\circ} 28^{\prime}$, Altitude $76 \mathrm{~m}$ ) of the Chinese Academy of Sciences, and from the trees of L. speciosa growing in the Xishuangbanna Tropical Botanical Garden (N $21^{\circ} 41^{\prime}$, E $101^{\circ} 25^{\prime}$, Altitude $570 \mathrm{~m}$ ), the Chinese Academy of Sciences. The fresh leaves from each accession were immediately dried with silica gel for further DNA extraction. Total genomic DNAs were extracted from each sample using the Plant Genomic DNA Kit (DP305) from Tiangen Biotech (Beijing) Co., Ltd., China.

\section{Chloroplast Genome Sequencing, Assembling, and Annotation}

The Lagerstroemia cp genomes were sequenced using the shortrange PCR (Polymerase Chain Reaction) method reported by Dong et al. (2012, 2013). The PCR protocol was as follows: preheating at $94^{\circ} \mathrm{C}$ for $4.5 \mathrm{~min}, 34$ cycles at $94^{\circ} \mathrm{C}$ for $50 \mathrm{~s}$, annealing at $55^{\circ} \mathrm{C}$ for $40 \mathrm{~s}$, and elongation at $72^{\circ} \mathrm{C}$ for 1.5 min, followed by a final extension at $72^{\circ} \mathrm{C}$ for $8 \mathrm{~min}$. PCR amplification was performed in an Applied Biosystems VeritiTM 96-Well Thermal Cycler (Model\#: 9902, made in Singapore). The amplified DNA fragments were sent to Shanghai Majorbio BioPharm Technology Co., Ltd (Beijing) for Sanger sequencing in both the forward and reverse directions using a 3730xl DNA analyzer (Applied Biosystems, Foster City, CA, USA). DNA regions containing poly structures or difficult to amplify were further sequenced using newly designed primers for confirming reliable and high quality sequencing results.

The cp DNA sequences were manually confirmed and assembled using Sequencher (v4.6) software, and cp genome annotation was performed using the Dual Organellar Genome Annotator (DOGMA; Wyman et al., 2004). BLASTX and BLASTN searches were employed to accurately annotate the protein-encoding genes and to identify the locations of the ribosomal RNA (rRNA) and transfer RNA (tRNA) genes. Gene annotation information from other closely related plant species was also utilized for confirmation when the boundaries of the exons or introns could not be precisely determined because of the limited power of BLAST in cp genome annotation. The cp genome map was drawn using Genome Vx software (Conant and Wolfe, 2008; Figure 1). The cp genome sequences have been deposited to GenBank with the following accession numbers: KF572028 for L. indica "Lüzhao Hongdie," KF572029 for L. subcostata and KX572149 for L. speciosa. The cp genome sequences of L. fauriei (KT358807), L. indica (KX263727), and L. guilinensis (KU885923) were downloaded from GenBank (https://www.ncbi.nlm.nih.gov). 


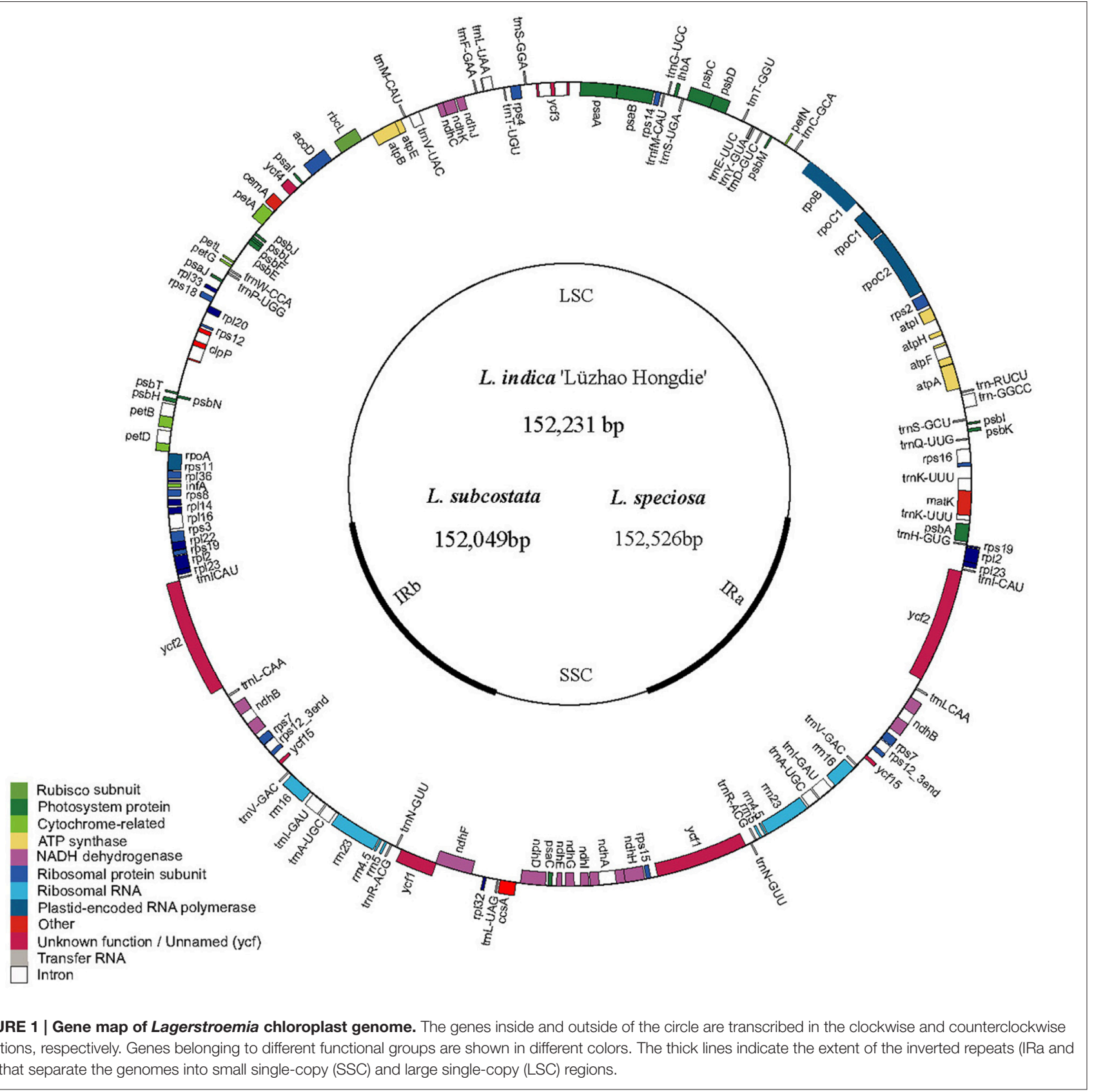

\section{Simple Sequence Repeat Analysis}

Perl script MISA (Thiel et al., 2003) was used to search for simple sequence repeat (SSRs or microsatellites) loci in the cp genomes. The minimum numbers (thresholds) of the SSRs were 10, 5, 4, 3, 3 , and 3 for mono-, di-, tri-, tetra-, penta-, and hexa-nucleotides, respectively. All of the repeats found were manually verified and redundant results were removed.

\section{Chloroplast Genome Analysis by Sliding Window}

These $\mathrm{cp}$ genome sequences were aligned using MAFFT (Katoh and Standley, 2013) and were manually adjusted using Se-Al 2.0 (Rambaut, 1996). We used two data sets (the sequence alignment of all the six complete Lagerstroemia cp genomes and the sequence alignment of five Lagerstroemia cp genomes excluding $L$. speciosa) for sliding window analysis, because of the high divergence of $L$. speciosa from the other five cp genomes (Figure 2). Sliding window analysis was conducted to generate nucleotide diversity $(\mathrm{Pi})$ of the cp genome using DnaSP (DNA Sequences Polymorphism version 5.10.01) software (Librado and Rozas, 2009). The step size was set to $200 \mathrm{bp}$, with a $600 \mathrm{bp}$ window length.

\section{Sequence Divergence Analysis}

The alignment of the six Lagerstroemia complete cp genome sequences was visualized using mVISTA program 


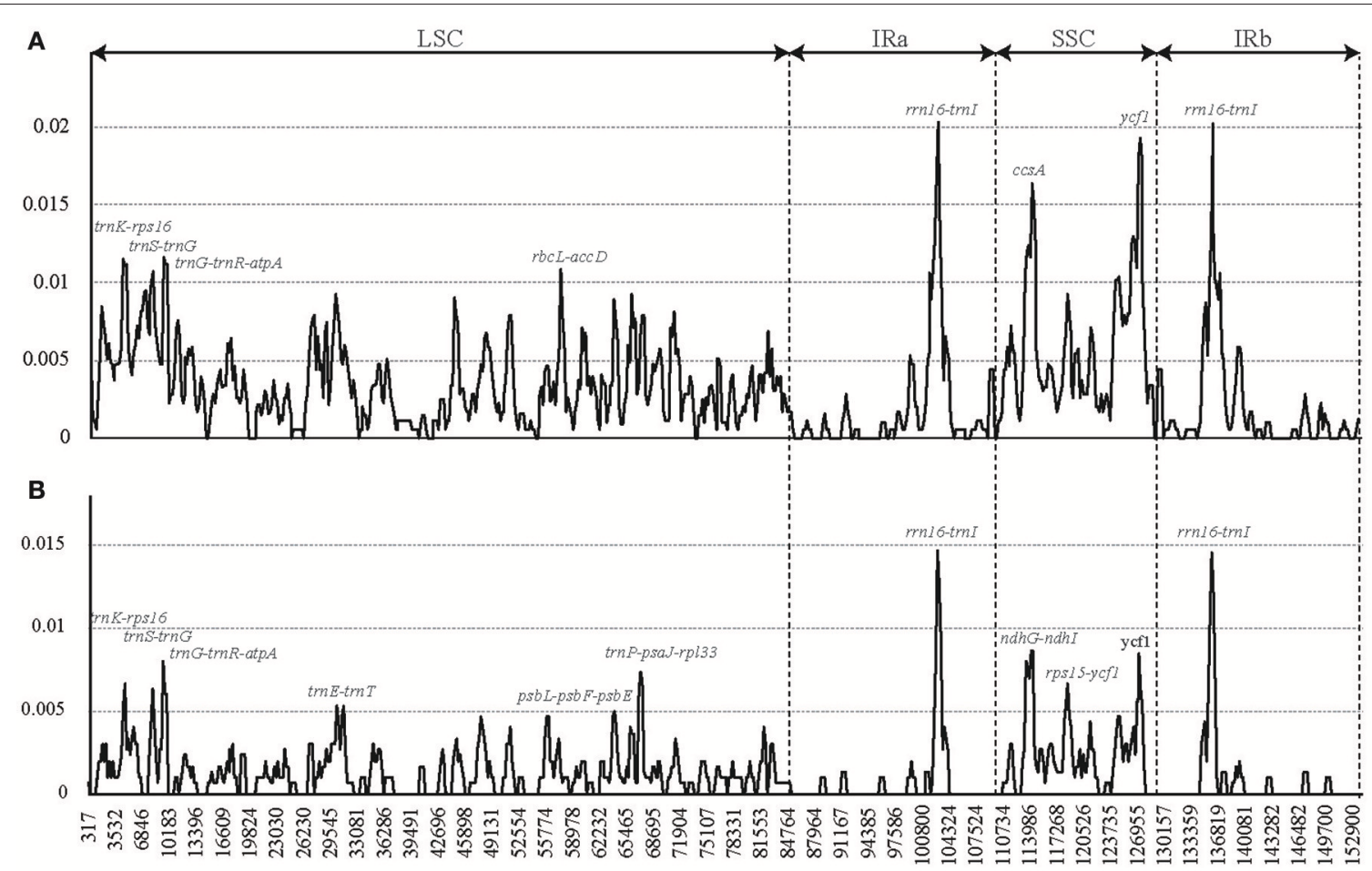

FIGURE 2 | Sliding window analysis of the whole chloroplast genomes of six Lagerstroemia taxa (A) and five Lagerstroemia taxa (not including L. speciosa) (B) (window length: $600 \mathrm{bp}$, step size: $200 \mathrm{bp}$ ). X-axis, position of the midpoint of a window; Y-axis, nucleotide diversity of each window.

in Shuffle-LAGAN mode (Frazer et al., 2004) in order to show inter- and intra-specific variations (Figure 3). Variable and parsimony-informative base sites across the complete cp genomes, and the large single copy (LSC), small single copy (SSC), and inverted repeats (IR) regions of the six cp genomes were calculated using Mega 6.0 software (Tamura et al., 2013). Insertions/deletions (indels) were manually detected using DnaSP software. To estimate selection pressures, nonsynonymous $(\mathrm{dN})$, and synonymous (dS) substitution rates of the combined sequences of 79 protein coding genes were calculated using PAML with the yn00 program (Yang, 2007).

\section{Phylogenetic Analysis}

Phylogenetic analysis was conducted using the complete chloroplast genome sequences of the six Lagerstroemia taxa mentioned above, with one Onagraceae species (Oenothera argillicola, 165,061 bp, GenBank accession No. EU262887) that was used as an outgroup (Figure 4).

Maximum parsimony (MP) analyses were conducted using PAUP v4b10 (Swofford, 2003). All characters were equally weighted, gaps were treated as missing, and character states were treated as unordered. Heuristic search was performed with MULPARS option, tree bisection-reconnection (TBR) branch swapping, and random stepwise addition with 1,000 replications. The Maximum likelihood (ML) analyses were performed using RAxML 8.0 (Stamatakis, 2006). For ML analyses, the best-fit model, general time reversible (GTR) $+\mathrm{G}$ was used in all analysis as suggested with 1,000 bootstrap replicates.
Bayesian inference (BI) was performed with Mrbayes v3.2 (Ronquist et al., 2012). The Markov chain Monte Carlo (MCMC) analysis was run for $2 \times 5,000,000$ generations. Trees were sampled at every 1,000 generations with the first $25 \%$ discarded as burn-in. The remaining trees were used to build a $50 \%$ majority-rule consensus tree. The stationarity was considered to be reached when the average standard deviation of split frequencies remained below 0.001 .

\section{RESULTS AND DISCUSSION}

\section{Chloroplast Genome Organization of the Lagerstroemia Taxa}

The nucleotide sequences of the six Lagerstroemia cp genomes ranged from $152,049 \mathrm{bp}$ (L. subcostata) to $152,526 \mathrm{bp}$ (L. speciosa) in length (Figure 1 and Table 1). The six Lagerstroemia cp genome sequences have minor differences in length (no more than 477 bp; Table 1). The average GC content was $37.59 \%$, which is almost identical with each other among the six complete Lagerstroemia cp genomes. When duplicated genes in IR regions were counted only once, the six Lagerstroemia cp genomes each identically harbored 112 different genes with the same arrangement order, including 78 protein-coding, 4 rRNA, and 30 tRNA genes (Figure 1, Table 1, and Table S1). The gene organization, gene order and GC content were highly identical and similar to those of other higher plants (Figure 1). The overall genomic structure including gene number and gene order were well-conserved. 


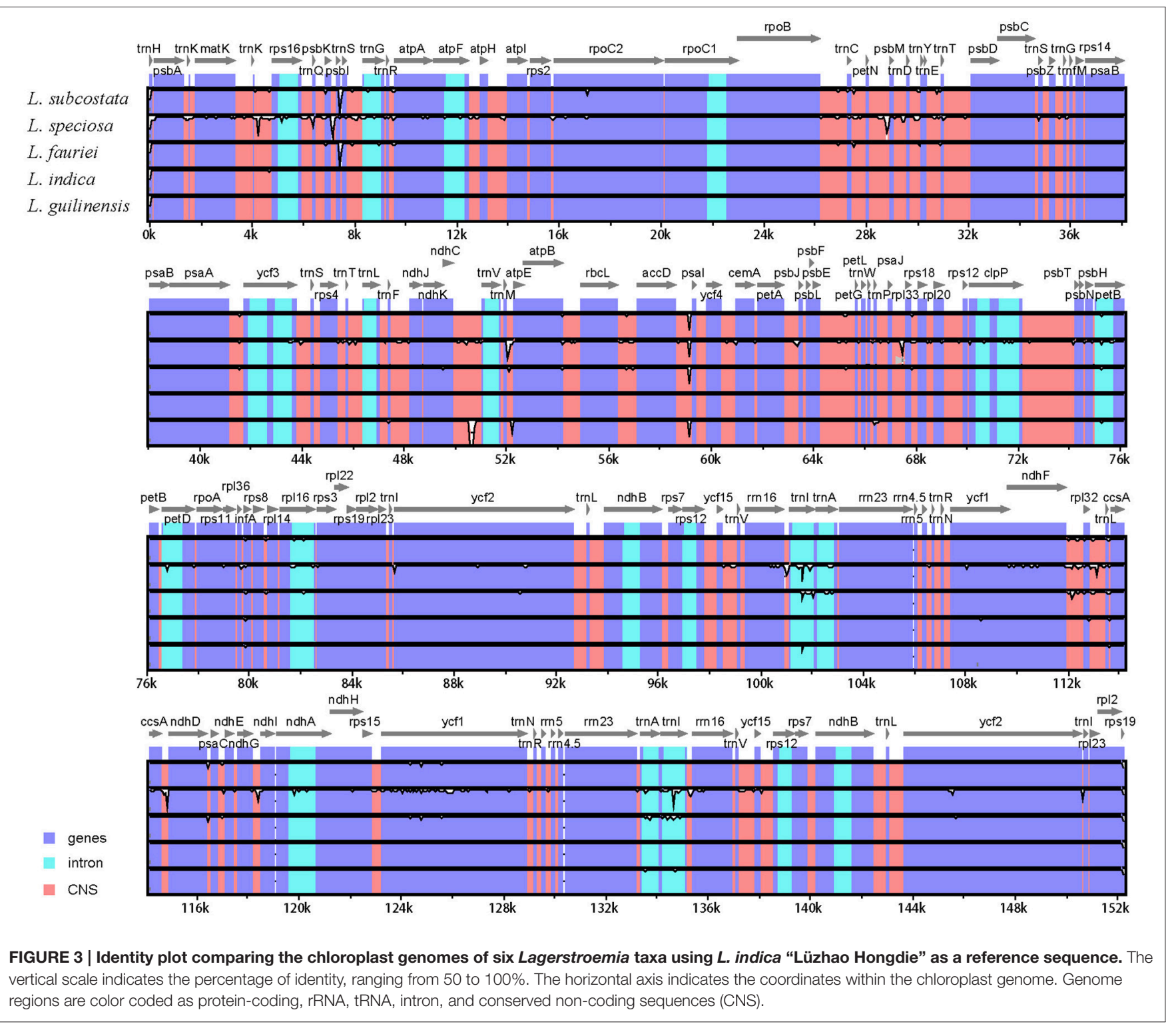

Although cp genomes are highly conserved in terms of genomic structure and size, the IR/SC junction position change caused by expansion and contraction of the IR/SC boundary regions was usually considered as a primary mechanism in creating the length variation of the higher plant cp genomes (Kim and Lee, 2005; Asaf et al., 2016; Dong et al., 2016; Yang et al., 2016; Zhang et al., 2016). In this study, however, the IR/SC junction position change was not observed among the six cp genomes. This indicated that the IR/SC junction is relatively conserved in Lagerstroemia in comparison with other plant groups, such as Quercus (Yang et al., 2016) and Epimedium (Zhang et al., 2016). Further, study is necessary by sampling more species of the genus across the world for confirmation.

The rpl2 intron loss was observed in the three newly sequenced Lagerstroemia cp genomes in this study. The occurrence of rpl2 intron loss in Lagerstroemia was considered to be one of the important evolutionary events in the Lythraceae of the rosids. It was inferred to occur after the divergence of the Lythraceae from the Onagraceae, but prior to the divergence of the Lythraceae genera (Gu et al., 2016a).

\section{SSR Analysis of the Lagerstroemia cp Genomes}

Simple sequence repeats (SSRs) in the cp genome can be highly variable at the intra-specific level, and are therefore often used as genetic markers in population genetics and evolutionary studies (Dong et al., 2013, 2016; Kaur et al., 2015; Suo et al., 2016; Yang et al., 2016). We analyzed the simple sequence repeats (SSRs) in the cp genomes (Tables 2, 3, Tables S2, S3). The lengths of SSRs ranged from 10 to 15 bp. Comparative analysis of the six Lagerstroemia cp genome sequences indicated that totally five categories of SSRs (mono-nucleotide, di-nucleotide, tri-nucleotide, tetranucleotide, and penta-nucleotide repeats) were detected, 


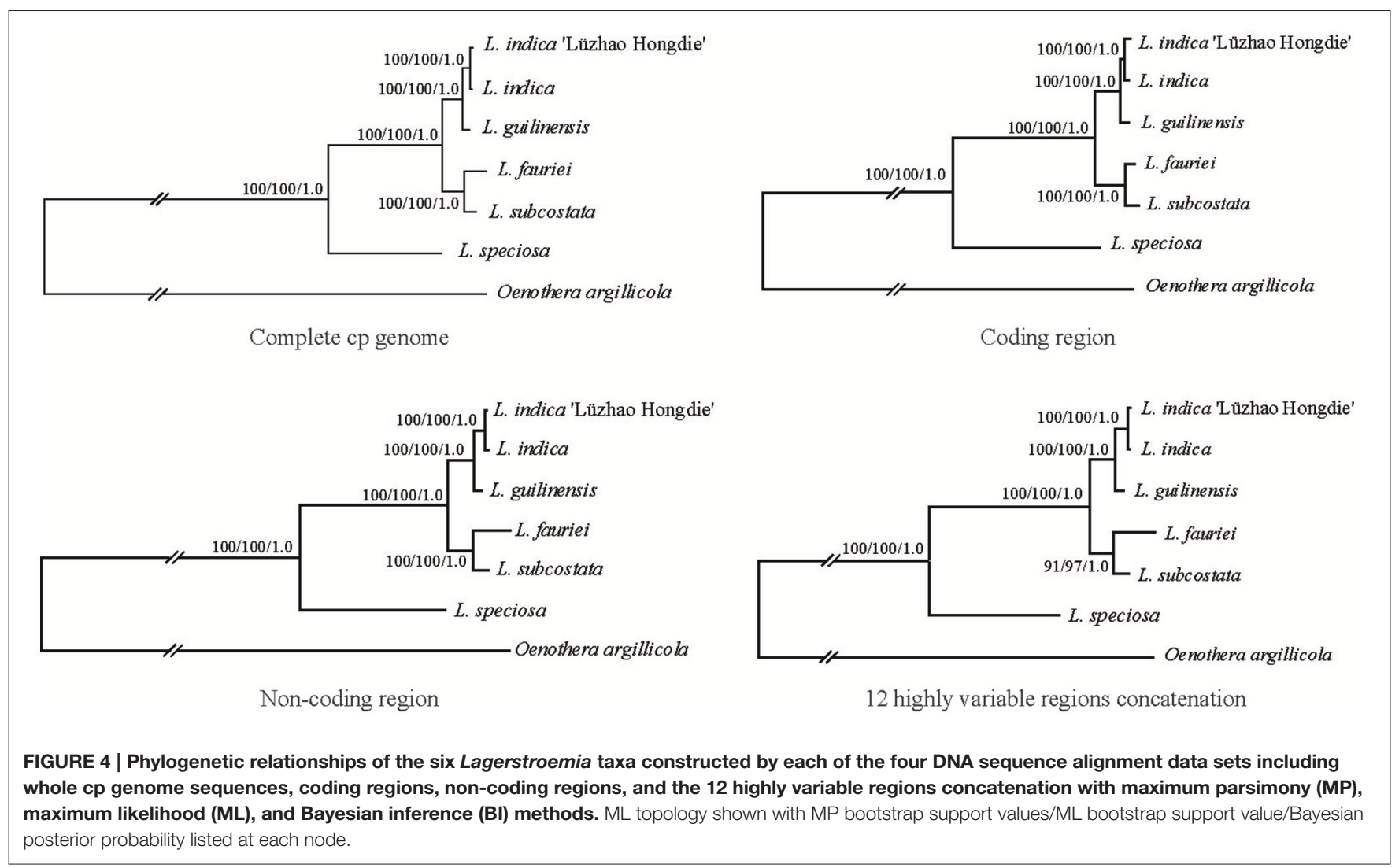

TABLE 1 | Summary of complete chloroplast genome features of the six Lagerstroemia taxa.

\begin{tabular}{|c|c|c|c|c|c|c|}
\hline & L. indica "Lüzhao Hongdie" & L. indica & L. subcostata & L. speciosa & L. fauriei & L. guilinensis \\
\hline Large single copy (LSC, bp) & 84,062 & 84,046 & 83,890 & 84,193 & 83,920 & 83,811 \\
\hline Small single copy (SSC, bp) & 16,919 & 16,915 & 16,909 & 16,833 & 16,934 & 16,909 \\
\hline Inverted repeat (IR, bp) & 25,625 & 25,622 & 25,625 & 25,750 & 25,793 & 25,677 \\
\hline Total & 152,231 & 152,205 & 152,049 & 152,526 & 152,440 & 152,074 \\
\hline rRNA & 4 & 4 & 4 & 4 & 4 & 4 \\
\hline tRNA & 30 & 30 & 30 & 30 & 30 & 30 \\
\hline Total & 112 & 112 & 112 & 112 & 112 & 112 \\
\hline GC\% & 37.59 & 37.59 & 37.59 & 37.57 & 37.60 & 37.62 \\
\hline
\end{tabular}

including 35 SSR types and 275 SSR loci. The most abundant were mono-nucleotide repeats, which accounted for $53.82 \%$ in the total, followed by tetra-nucleotide repeats (16.36\%), tri-nucleotide repeats $(14.91 \%)$, and di-nucleotides repeats (10.55\%), subsequently. Penta-nucleotide repeats had the least amount (4.36\%; Tables 2, 3, Tables S2, S3). In Quercus species, mononucleotide repeats are the most abundant, accounting for about $80 \%$ of the total SSRs (Yang et al., 2016). In the cp genome of Dianthus, homopolymers were most common, accounting for 95.58\% of the SSRs (Raman and Park, 2015). These results suggest that mononucleotide repeats may contribute more to the genetic variations in comparison with other SSRs. The SSR information will be important for understanding the genetic diversity status of the global Lagerstroemia plants.

In this study, these 275 SSRs were mainly located in intergenic spacers (161 SSRs, 58.55\%) or LSC region (183 SSRs, 66.55\%), only a minority (IRa: 22 SSRs, 8.00\%; IRb: 23 SSRs, 8.36\%) of SSRs were located in the IR regions. Sixty-three SSRs $(22.91 \%)$ were located in eight gene (CDS) regions ( $r p o A, r p o B, r p o C 2$, cemA, $n d h D, n d h F, y c f 1, y c f 2$; Tables 2, 3, Tables S2, S3). Fifty-four SSRs (19.64\%) were located in intron regions. The 
TABLE 2 | Distribution of each SSR category in the six Lagerstroemia cp genomes.

\begin{tabular}{|c|c|c|c|c|c|c|c|c|c|}
\hline & Category & Number & Intergenic & Gene & Intron & LSC & SSC & IRa & IRb \\
\hline \multirow[t]{6}{*}{ L. fauriei } & Mono-nucleotide & 28 & 18 & 4 & 6 & 28 & 6 & 2 & 2 \\
\hline & Di-nucleotide & 4 & 1 & 2 & 1 & 3 & 1 & 0 & 0 \\
\hline & Tri-nucleotide & 6 & 4 & 2 & 1 & 3 & 2 & 1 & 1 \\
\hline & Tetra-nucleotide & 7 & 3 & 3 & 2 & 6 & 2 & 0 & 0 \\
\hline & Penta-nucleotide & 2 & 2 & 0 & 0 & 0 & 0 & 1 & 1 \\
\hline & Subtotal & 47 & 28 & 11 & 10 & 40 & 11 & 4 & 4 \\
\hline \multirow[t]{6}{*}{ L. guilinensis } & Mono-nucleotide & 24 & 17 & 4 & 3 & 16 & 5 & 2 & 2 \\
\hline & Di-nucleotide & 4 & 2 & 2 & 1 & 4 & 1 & 0 & 0 \\
\hline & Tri-nucleotide & 7 & 4 & 2 & 1 & 3 & 2 & 1 & 1 \\
\hline & Tetra-nucleotide & 7 & 2 & 3 & 2 & 5 & 2 & 0 & 0 \\
\hline & Penta-nucleotide & 2 & 2 & 0 & 0 & 0 & 0 & 1 & 1 \\
\hline & Subtotal & 44 & 27 & 11 & 7 & 28 & 10 & 4 & 4 \\
\hline \multirow[t]{6}{*}{ L. indica } & Mono-nucleotide & 18 & 10 & 3 & 5 & 11 & 5 & 1 & 1 \\
\hline & Di-nucleotide & 6 & 4 & 1 & 1 & 4 & 1 & 1 & 0 \\
\hline & Tri-nucleotide & 7 & 4 & 2 & 1 & 3 & 2 & 1 & 1 \\
\hline & Tetra-nucleotide & 7 & 2 & 3 & 2 & 5 & 2 & 0 & 0 \\
\hline & Penta-nucleotide & 2 & 2 & 0 & 0 & 0 & 0 & 1 & 1 \\
\hline & Subtotal & 40 & 22 & 9 & 9 & 23 & 10 & 4 & 3 \\
\hline \multirow[t]{6}{*}{ L. indica "Lüzhao Hongdie" } & Mono-nucleotide & 29 & 19 & 4 & 6 & 18 & 7 & 2 & 2 \\
\hline & Di-nucleotide & 5 & 3 & 2 & 0 & 4 & 1 & 0 & 0 \\
\hline & Tri-nucleotide & 7 & 4 & 2 & 1 & 3 & 2 & 1 & 1 \\
\hline & Tetra-nucleotide & 7 & 2 & 3 & 2 & 5 & 2 & 0 & 0 \\
\hline & Penta-nucleotide & 2 & 2 & 0 & 0 & 0 & 0 & 1 & 1 \\
\hline & Subtotal & 50 & 30 & 11 & 9 & 30 & 12 & 4 & 4 \\
\hline \multirow[t]{6}{*}{ L. speciosa } & Mono-nucleotide & 24 & 17 & 2 & 5 & 20 & 2 & 1 & 1 \\
\hline & Di-nucleotide & 6 & 3 & 2 & 1 & 4 & 2 & 0 & 0 \\
\hline & Tri-nucleotide & 7 & 4 & 2 & 1 & 4 & 1 & 1 & 1 \\
\hline & Tetra-nucleotide & 9 & 4 & 3 & 2 & 7 & 2 & 0 & 0 \\
\hline & Penta-nucleotide & 2 & 2 & 0 & 0 & 0 & 0 & 1 & 1 \\
\hline & Subtotal & 48 & 30 & 9 & 9 & 35 & 7 & 3 & 3 \\
\hline \multirow[t]{7}{*}{ L. subcostata } & Mono-nucleotide & 25 & 15 & 4 & 6 & 15 & 6 & 2 & 2 \\
\hline & Di-nucleotide & 4 & 1 & 2 & 1 & 3 & 1 & 0 & 0 \\
\hline & Tri-nucleotide & 7 & 4 & 2 & 1 & 3 & 2 & 1 & 1 \\
\hline & Tetra-nucleotide & 8 & 3 & 4 & 1 & 6 & 2 & 0 & 0 \\
\hline & Penta-nucleotide & 2 & 1 & 0 & 1 & 0 & 0 & 1 & 1 \\
\hline & Subtotal & 46 & 24 & 12 & 10 & 27 & 11 & 4 & 4 \\
\hline & Total & 275 & 161 & 63 & 54 & 183 & 61 & 23 & 22 \\
\hline
\end{tabular}

distribution of SSRs is variable significantly among the four regions in each of the six Lagerstroemia cp genomes, which is identical with previous reports (Dong et al., 2016; Yang et al., 2016).

Among the 148 homopolymer SSRs of the six Lagerstroemia cp genomes, $141(95.27 \%)$ are the A/T type, distributed mostly in intergenic (90 A/T loci, 63.83\%) and LSC (102 A/T loci,
72.34\%) regions (Tables S2, S3). In Nicotiana otophora, all mono-nucleotides (100\%) are composed of A/T (Asaf et al., 2016). In the five Epimedium cp genomes, mono-nucleotide SSRs were found to be the richest, up to $72.76 \%$, and the mono-nucleotide $\mathrm{A} / \mathrm{T}$ repeat units occupied $80.17 \%$ in the homopolymer SSRs. Our results are identical with the observation that the occurrence of transversion substitutions is 
correlated to some extent with high A/T content regions of the cp genome (Morton and Clegg, 1995; Morton et al., 1997).

In the cp genomes of five Quercus species, most of the repeat units were distributed in intergenic or intron regions, and only a minority were located in gene regions $(y c f 1, y c f 2, p s a A, p s a B$, trnS-GCU, trnS-UGA, trnG-GCC, trnG-UCC, trnS-UGA, and trnS-GGA; Yang et al., 2016).

In this study, no variation was detected in the repeat number of penta-nucleotide repeat category and only minor variation was observed in the repeat number of tri-nucleotide repeat category among species and/or cultivars. The repeat numbers of mono-nucleotide, di-nucleotide and tetra-nucleotide repeat categories were found variable significantly among the six cp genomes. Mono-nucleotide repeat category is the dominant variation source, especially between cultivars rather than between species, e.g., with 29 in L. indica "Lüzhao Hongdie," and 18 in L. indica (Tables 2, 3, Tables S2, S3).

In the five Epimedium cp genomes, the detected 116 SSR loci mainly located in intergenic spacers (IGS, 62.07\%), followed by introns (23.28\%) and CDS (13.79\%) regions. These are similar with our results. It was observed that 16 SSRs were located in 10 protein-coding genes $(r p o C 2, r p o B, p s b C, p s a A, p s b F, y c f 1$, $y c f 2, r p l 32, n d h E$, and $n d h H)$ of the five Epimeidium cp genomes (Zhang et al., 2016). Therefore, evidences strongly suggest that the occurrence and genetic variations of SSRs in genes (such as, $y c f 1$ ) may have phylogenetic significance. This is worth further study in the future.

A preference for occurrence of SSRs in intergenic or gene regions was observed between plant families and among the samples/taxa within family. The cp SSRs of the six Lagerstroemia taxa represented abundant variation, and are useful for detecting genetic polymorphisms at population, intraspecific, and cultivar levels as well as comparing more distant phylogenetic relationships among Lagerstroemia species.

\section{Genome Sequence Divergence among the Lagerstroemia Species/Cultivars}

We used mVISTA to perform a sequence identity analysis, with L. indica "Lüzhao Hongdie" as a reference (Figure 3).
The alignment revealed high sequence similarity across the cp genomes, which suggests that they are highly conserved. Non-coding and SC regions exhibit higher divergence levels than coding and IR regions, respectively.

The LSC and SSC regions contributed 150 and 55 informative base sites, respectively, while the IR regions contributed only 15 informative sites (Table 4). The SSC region showed the highest nucleotide diversity (0.00639), followed by the LSC region (0.00345) and the IR region (0.00175; Table 4). Lagerstroemia speciosa presented the highest numbers of nucleotide substitutions and insertions/deletions (indels) among the six Lagerstroemia taxa, while the nucleotide diversity, and the numbers of nucleotide substitutions and insertions/deletions (indels) at cultivar level were found to be the smallest (Tables 4, 5).

Pairwise substitution rates $(\mathrm{dN} / \mathrm{dS})$ between the Lagerstroemia cp genomes were calculated based on the 78 protein-coding gene sequences (Table 6). The numbers of nucleotide substitutions and indels varied from 29 to 315, and 24 to 1089 , respectively (Table 5). There were always fewer $\mathrm{dN}$ than $\mathrm{dS}$. The $\mathrm{dN} / \mathrm{dS}$ ratio ranged from 0.1688 to 0.6081 . The highest $\mathrm{dN} / \mathrm{dS}$ ratio occurred between $L$. indica and L. guilinensis. The lowest $\mathrm{dN} / \mathrm{dS}$ ratio occurred between Lagerstroemia indica and L. indica "Lüzhao Hongdie" (Table 6). In our study, the $\mathrm{dN} / \mathrm{dS}$ ratio is below 1 , indicating that the related gene regions might be under negative selection.

TABLE 4 | Variable site analyses in the six Lagerstroemia cp genomes.

\begin{tabular}{lcccc}
\hline & $\begin{array}{c}\text { Number of } \\
\text { sites }\end{array}$ & $\begin{array}{c}\text { Number of } \\
\text { variable } \\
\text { sites }\end{array}$ & $\begin{array}{c}\text { Number of } \\
\text { informative } \\
\text { sites }\end{array}$ & $\begin{array}{c}\text { Nucleotide } \\
\text { diversity }\end{array}$ \\
\hline Large single copy region & 84,868 & $771\left(0.91 \%^{\star}\right)$ & $150\left(19.46 \%{ }^{* *}\right)$ & 0.00345 \\
Small single copy region & 17,077 & $281(1.65 \%)$ & $55(19.57 \%)$ & 0.00639 \\
Inverted repeat region & 25,961 & $133(0.51 \%)$ & $15(11.28 \%)$ & 0.00175 \\
Complete cp genome & 153,842 & $1330(0.86 \%)$ & $238(17.89 \%)$ & 0.00322 \\
\hline
\end{tabular}

${ }^{\star}$ The percentage of variable sites in the number of sites. ${ }^{\star \star}$ The percentage of informative sites in the number of variable sites.

TABLE 3 | Numbers and percentage of SSRs in the six Lagerstroemia cp genomes.

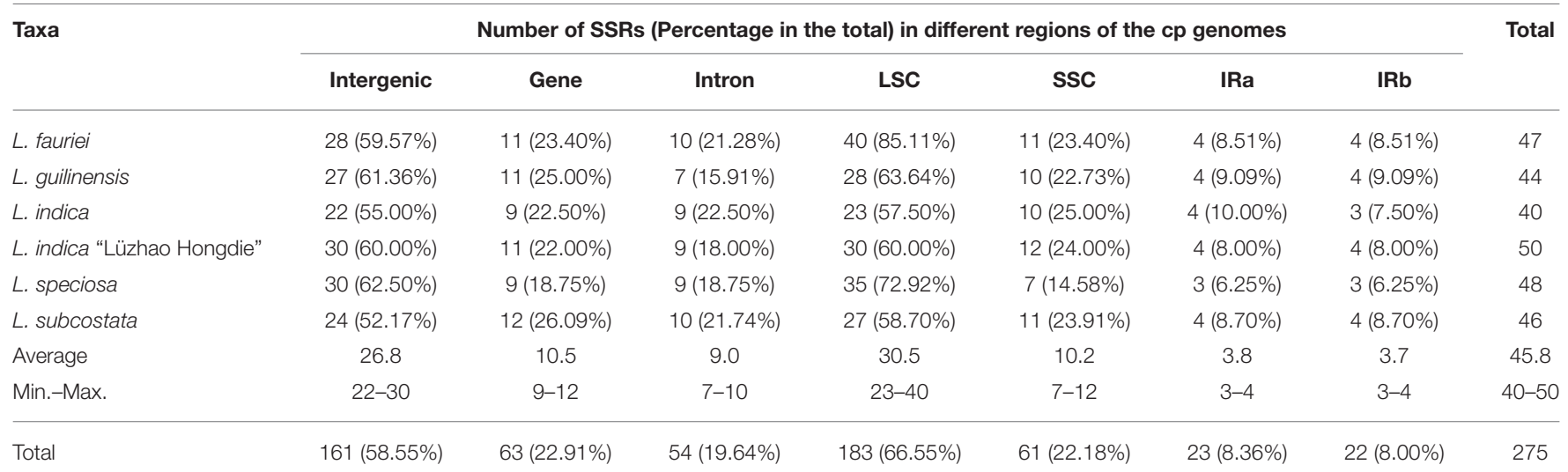

SSRs, simple sequence repeats. LSC, Large single copy region; SSC, Small single copy region; Ira, Inverted repeat region a; IRb, Inverted repeat region b. 
TABLE 5 | Number of nucleotide substitutions and insertions/deletions in the six Lagerstroemia complete cp gemomes.

\begin{tabular}{|c|c|c|c|c|c|c|}
\hline & L. indica "Lüzhao Hongdie” & L. subcostata & L. speciosa & L. fauriei & L. indica & L. guilinensis \\
\hline L. indica "Lüzhao Hongdie" & & 66 & 293 & 95 & 29 & 31 \\
\hline L. subcostata & 257 & & 295 & 57 & 79 & 72 \\
\hline L. speciosa & 1084 & 1089 & & 315 & 297 & 301 \\
\hline L. fauriei & 309 & 134 & 1105 & & 103 & 91 \\
\hline L. indica & 24 & 249 & 1082 & 303 & & 44 \\
\hline L. guilinensis & 63 & 254 & 1083 & 291 & 57 & \\
\hline
\end{tabular}

The lower triangle indicates the number of nucleotide substitutions, the upper triangle shows the number of insertions/deletions.

TABLE 6 | Pairwise substitution rates (dN/dS) between the Lagerstroemia chloroplast genomes based on the 78 protein-coding gene sequences.

\begin{tabular}{|c|c|c|c|c|c|}
\hline No. & L. indica "Lüzhao Hongdie" & L. subcostata & L. speciosa & L. fauriei & L. indica \\
\hline \multicolumn{6}{|c|}{ L. indica "Lüzhao Hongdie" } \\
\hline L. subcostata & $0.3102(0.0009 / 0.0029)$ & & & & \\
\hline L. speciosa & $0.3762(0.0036 / 0.0095)$ & $0.3755(0.0037 / 0.0098)$ & & & \\
\hline L. fauriei & $0.3178(0.0009 / 0.0027)$ & $0.2605(0.0002 / 0.0009)$ & $0.3710(0.0036 / 0.0097)$ & & \\
\hline L. indica & $0.1688(0.0001 / 0.0006)$ & $0.3374(0.0010 / 0.0028)$ & $0.3767(0.0036 / 0.0094)$ & $0.3326(0.0009 / 0.0027)$ & \\
\hline
\end{tabular}

We chose the 12 relatively highly variable regions including 2 gene regions and 10 intergenic regions from the cp genomes that might be undergoing a more rapid nucleotide substitution at species and cultivar levels, as potential molecular markers for application in phylogenetic analyses and plant identification in Lagerstroemia (Figure 2, Table 7). They are trnK-rps16, trnStrnG, $\operatorname{trn} G$-trnR-atpA, trnE-trnT, $r b c L-a c c D, p s b L-p s b F-p s b E$, trnP-psaJ-rpl33, rrn16-trnI, ccsA, ndhG-ndhI, rps15-ycf1, and $y c f 1$. Primers for these regions are shown in Table 7. Yang et al. (2016) determined five most variable coding regions and 14 most variable non-coding regions as potential molecular markers for Quercus germplasm resources, which are identical with the variable regions found in Lagerstroemia, except for trnEtrnT, psbL-psbF-psbE, trnP-psaJ-rpl33, ndhG-ndhI, and rps15$y c f 1$. Further, study is expected to utilize these cp DNA markers in global detection of the Lagerstroemia germplasm resources.

\section{Phylogenetic Analysis}

Phylogenetic analysis using cp genome sequences have resolved numerous lineages within the flowering plants (Jansen et al., 2007; Moore et al., 2007). The cp DNA regions of atpF-atpH, $m a t K, p s b K-p s b I, r b c L$, and $t r n H-p s b A$ have been recommended and used as species-level barcodes with a great success (Suo et al., 2012, 2015, 2016; Dong et al., 2015, 2016). However, these five cp DNA markers are not powerful enough when closely related species or cultivars are under considerations. Therefore, genomic comparative researches of more complete cp genome sequences have become necessary.

In this study, all of the six Lagerstroemia taxa were discriminated completely with high bootstrap support based on each of the four DNA sequence alignment data sets including whole cp genome sequences, coding regions, non-coding regions, and the 12 highly variable regions concatenation using maximum parsimony (MP), maximum likelihood (ML), and Bayesian inference (BI) methods (Figure 4). L. guilinensis, L. indica "Lüzhao Hongdie," and L. indica showed a very close genetic relationship. The six taxa were separated into three evolutionary branches. The branch including L. subcostata and L. fauriei was a sister to the branch containing L. guilinensis, L. indica "Lüzhao Hongdie," and L. indica. L. speciosa was placed at the basal position, and showed a large divergence from the rest five Lagerstroemia taxa. A better resolution was obtained by the sequence data set from the non-coding regions as compared to each of the other three datasets. Similar resolution can be obtained using a sequence data set from 12 highly variable $\mathrm{cp}$ regions with lower cost.

\section{CONCLUSIONS}

This study reports the comparative analysis results of six Lagerstroemia cp genome sequences with detailed gene annotation. The six cp genomes are similar in structure and have a high degree of the synteny of gene order. The IR/SC junction position change was not observed among the six $\mathrm{cp}$ genomes, indicating that the IR/SC junction is relatively conservative in Lagerstroemia in comparison with other plant groups, such as Quercus and Epimedium. Further study is necessary for confirmation within the whole genus by sampling more species. Twelve cp DNA markers were developed from the relatively highly variable regions. All of the six Lagerstroemia taxa were discriminated completely with high bootstrap support based on each of the four DNA sequence alignment data sets including whole $\mathrm{cp}$ genome sequences, coding regions, noncoding regions, and 12 highly variable regions using maximum parsimony (MP), maximum likelihood (ML), and Bayesian inference (BI) methods. A better resolution was obtained by the 
TABLE 7 | Primers for PCR amplification of the 12 relatively highly variable regions among the six Lagerstroemia taxa.

\begin{tabular}{|c|c|c|c|c|c|}
\hline No. & Region amplified & Forward primer $\left(5^{\prime} \rightarrow 3^{\prime}\right)$ & Reverse primer $\left(5^{\prime} \rightarrow 3^{\prime}\right)$ & Size (bp) & Annealing temperature $\left({ }^{\circ} \mathrm{C}\right)$ \\
\hline 1 & trnK-rps 16 & TGGGTTCATAGGACTCTATCCA & TTGCAATTGATGTGCGATCTCGA & 1202 & 56 \\
\hline 2 & $\operatorname{trnS-trnG}$ & ACCGAGTTATCAACGGAAACGGA & TAAAGTITCTGCTCGGAATAAGA & 882 & 53 \\
\hline 3 & trnG-trnR-atpA & TCTAGAGGGATTATCTAGAAAGCA & AAGAGGTCAACGATTACGTGAGT & 975 & 55 \\
\hline 4 & $\operatorname{trn} \mathrm{E}-\operatorname{trn} \mathrm{T}$ & AGAGGAATGTCCGTTGGG & CGATGACTTACGCCTTACC & 1471 & 53 \\
\hline 5 & $r b c L-a c c D$ & TCTCTTAATTGAATTGCAATTCA & AATAGATGAATAGTCATTCGATGA & 703 & 49.5 \\
\hline 6 & $p s b L-p s b F-p s b E$ & GTGATCCTTCCGAATGGGATAAG & CAGTGAATTTCCATITACTGATAT & 672 & 51.8 \\
\hline 7 & trnP-psaJ-rp/33 & AGTAGAAGGTITATATATCTAATA & GATTATITCGTTGCAATCACAAC & 905 & 48.5 \\
\hline 8 & $r r n 16-t r n \mid$ & TTAGTTGCCACCGGTATGAGAGT & GGTCCTCTTCCCCATTACTTAGA & 1845 & 58 \\
\hline 9 & $\operatorname{ccs} A$ & AGGTATAATCCATGAATATTGAT & TGAATTCATTATAGGACTTATTA & 1755 & 48 \\
\hline 10 & ndhG-ndhl & ATCGGTTGATAAATGAATTCCAA & CAAGGTTCAATITGATCTAATCT & 790 & 51 \\
\hline 11 & rps15-ycf1 & TAAGTCTTCGTATCTTATTGGTG & GAGTTTGGATATTCTGATGATTCA & 1122 & 53 \\
\hline 12 & $y c f 1$ & TAACCTCAGCCTTAGCATT & GGACAGAATAGACAAACCCT & 2191 & 50 \\
\hline
\end{tabular}

sequence data set from the non-coding regions rather than by each of the other three data sets, with no significant difference among the analytic methods. Similar resolution result can be obtained by the sequence data set from 12 highly variable regions with lower cost. The six taxa were separated into three evolutionary branches. The branch including L. subcostata and L. fauriei is a sister to branch formed by L. guilinensis, L. indica "Lüzhao Hongdie," and L. indica. L. speciosa alone was placed at the basal position, and showed a large divergence from the rest five Lagerstroemia taxa. The data presented here will facilitate the understanding of the evolutionary history of crape myrtles. These findings provide an informative and valuable genetic source of the Lagerstroemia germplasm resources for identifying species, elucidating taxonomy, and reconstructing the phylogeny of the Lagerstroemia genus.

\section{AUTHOR CONTRIBUTIONS}

CX performed the experiments, analyzed the data, contributed reagents/materials/analysis tools, wrote the paper, reviewed drafts of the paper. WD conceived and designed the experiments, performed the experiments, analyzed the data, wrote the paper, prepared figures and/or tables, reviewed drafts of the paper. WL, YL, XX conceived and designed the experiments, contributed reagents/materials/analysis tools, wrote the paper, reviewed drafts of the paper. JS, KH contributed reagents/materials/analysis tools, reviewed drafts of the paper. XJ

\section{REFERENCES}

APG III (2009). An update of the Angiosperm Phylogeny Group classification for the orders and families of flowering plants: APG III. Bot. J. Linnean Soc. 161, 105-121. doi: 10.1111/j.1095-8339.2009.00996.x

Asaf, S., Khan, A. L., Khan, A. R., Waqas, M., Kang, S. M., Khan, M. A., et al. (2016). Complete chloroplast genome of Nicotiana otophora and its comparison with related species. Front. Plant Sci. 7:843. doi: 10.3389/fpls.2016.00843

Conant, G. C., and Wolfe, K. H. (2008). GenomeVx: simple web-based creation of editable circular chromosome maps. Bioinformatics 24, 861-862. doi: 10.1093/bioinformatics/btm598 wrote the paper, reviewed drafts of the paper. ZS conceived and designed the experiments, performed the experiments, analyzed the data, contributed reagents/materials/analysis tools, wrote the paper, reviewed drafts of the paper.

\section{FUNDING}

The study was financially supported by "Collection, Conservation, and Evaluation of Forest Tree Germplasm Resources" (LKZ201496-1-3) of Shandong Provincial Agricultural Elite Varieties Project, the joint projects No. 70009C1036 and 70009C1020, the National Natural Science Foundation of China (No. 30972412), and the National Forest Genetic Resources Platform (2005DKA21003).

\section{ACKNOWLEDGMENTS}

The authors thank Prof. Boxing Hou and Prof. Jin Chen and Cuihua $\mathrm{Gu}$ for advice and helpful discussion, and Shouzhou Zhang, Jun-jie Yu, Zulin Ning, and Bingqiang Xu for help in field investigation.

\section{SUPPLEMENTARY MATERIAL}

The Supplementary Material for this article can be found online at: http://journal.frontiersin.org/article/10.3389/fpls.2017. 00015/full\#supplementary-material 
Dong, W. P., Xu, C., Cheng, T., Lin, K., and Zhou, S. L. (2013). Sequencing angiosperm plastid genomes made easy: a complete set of universal primers and a case study on the phylogeny of Saxifragales. Genome Biol. Evol. 5, 989-997. doi: $10.1093 /$ gbe/evt 063

Dong, W. P., Xu, C., Li, C. H., Sun, J. H., Zuo, Y. J., Shi, S., et al. (2015).cf1, the most promising plastid DNA barcode of land plants. Sci. Rep. 5:8348. doi: $10.1038 /$ srep08348

Dong, W. P., Xu, C., Li, D. L., Jin, X. B., Lu, Q., and Suo, Z. L. (2016). Comparative analysis of the complete chloroplast genome sequences in psammophytic Haloxylon species (Amaranthaceae). Peer J. 4:e2699. doi: 10.7717/peerj.2699

Downie, S. R., and Jansen, R. K. (2015). A comparative analysis of whole plastid genomes from the Apiales: expansion and contraction of the inverted repeat, mitochondrial to plastid transfer of DNA, and identification of highly divergent noncoding regions. Syst. Bot. 40, 336-351. doi: 10.1600/036364415X686620

Frazer, K. A., Pachter, L., Poliakov, A., Rubin, E. M., and Dubchak, I. (2004). VISTA: computational tools for comparative genomics. Nucleic Acids Res. 32, W273-W279. doi: 10.1093/nar/gkh458

Graham, S. A., Hall, J., Sytsma, K., and Shi, S. (2005). Phylogenetic analysis of the Lythraceae based on four gene regions and morphology. Int. J. Plant Sci. 166, 995-1017. doi: 10.1086/432631

Gu, C. H., Tembrock, L. R., Zhang, D., and Wu, Z. Q. (2016b). Characterize the complete chloroplast genome of Lagerstroemia floribunda (Lythraceae), a narrow endemic crape myrtle native to Southeast Asia. Conserv. Genet. Resour. doi: 10.1007/s12686-016-0628-6

Gu, C. H., Tembrock, L. R., Johnson, N. G., Simmons, M. P., and Wu, Z. (2016a). The complete plastid genome of Lagerstroemia fauriei and loss of rpl2 intron from Lagerstroemia (Lythraceae). PLoS ONE 11:e0150752. doi: 10.1371/journal.pone.0150752

He, D., Liu, Y., Cai, M., Pan, H. T., and Zhang, Q. X. (2014). The first genetic linkage map of crape myrtle (Lagerstroemia) based on amplification fragment length polymorphisms and simple sequence repeats markers. Plant Breed. 133, 138-144. doi: 10.1111/pbr.12100

Huang, J. M., Hou, B. X., and Suo, Z. L. (2013a). Study on the Lagerstroemia indica cultivars in Shaoyang city I. J. Agr. 3, 47-53. Available online at: http://www. caaj.org/cjas/ch/reader/view_abstract.aspx?

Huang, J. M., Hou, B. X., and Suo, Z. L. (2013b). Study on the Lagerstroemia indica cultivars in Shaoyang city II. J. Agr. 3, 35-41. Avaiable online at: http://www. caaj.org/cjas/ch/reader/view_abstract.aspx?

Huang, J. M., Hou, B. X., and Suo, Z. L. (2013c). Study on the Lagerstroemia indica cultivars in Shaoyang city III. J. Agr. 3, 34-41. Available online at: http://www. caaj.org/cjas/ch/reader/view_abstract.aspx?

Huang, Y. L., and Shi, S. H. (2002). Phylogenetics of Lythraceae sensu lato: a preliminary analysis based on chloroplast rbcL gene, psaA-ycf3 spacer, and nuclear rDNA internal transcribed spacer (ITS). Int. J. Plant Sci. 163, 215-225. doi: $10.1086 / 338392$

Jansen, R. K., Cai, Z. Q., Raubeson, L. A., Daniell, H., dePamphilis, C. W., LeebensMack, J., et al. (2007). Analysis of 81 genes from 64 plastid genomes resolves relationships in angiosperms and identifies genome scale evolutionary patterns. Proc. Natl. Acad. Sci. U.S.A. 104, 19369-19374. doi: 10.1073/pnas.0709121104

Katoh, K., and Standley, D. M. (2013). MAFFT multiple sequence alignment software version 7: improvements in performance and usability. Mol. Biol. Evol. 30, 772-780. doi: 10.1093/molbev/mst010

Kaur, S., Panesar, P. S., Bera, M. B., and Kaur, V. (2015). Simple sequence repeat markers in genetic divergence and marker-assisted selection of rice cultivars: A review. Crit. Rev. Food Sci. Nutr. 55, 41-49. doi: 10.1080/10408398.2011.646363

Kim, K. J., and Lee, H. L. (2005). Wide spread occurrence of small inversions in the chloroplast genomes of land plants. Mol. Cells 19, 104-113. Available online at: https://www.baidu.com/link?url=_noO3HXu9B7KSoCqEjSECCzJckjU3Owgk NNvQT3sPw7_wr-95HX5cri0P9UPrPePJpQMe69A_Isr-XWzxXa77jeYNdL0L a5CPmpgck-_ZqGNiaUFEwPJkPNOoH4H1AFQ\&wd=\&eqid=9cda33380000a $59 \mathrm{~b} 000000035873 \mathrm{clbd}$

Librado, P., and Rozas, J. (2009). DnaSP v5: a software for comprehensive analysis of DNA polymorphism data. Bioinformatics 25, 1451-1452. doi: 10.1093/bioinformatics/btp187

Moore, M. J., Bell, C. D., Soltis, P. S., and Soltis, D. E. (2007). Using plastid genomescale data to resolve enigmatic relationships among basal angiosperms. Proc. Natl. Acad. Sci. U.S.A. 104, 19363-19368. doi: 10.1073/pnas.0708072104
Morton, B. R., and Clegg, M. T. (1995). Neighboring base composition is strongly correlated with base substitution bias in a region of the chloroplast genome. J. Mol. Evol. 41, 597-603. doi: 10.1007/BF00175818

Morton, B. R., Oberholzer, V. M., and Clegg, M. T. (1997). The influence of specific neighboring bases on substitution bias in noncoding regions of the plant chloroplast genome. J. Mol. Evol. 45, 227-231. doi: 10.1007/PL00006224

Pounders, C., Rinehart, T., and Sakhanokho, H. (2007). Evaluation of interspecific hybrids between Lagerstroemia indica and $L$. speciosa. HortScience 42 , 1317-1322. Available online at: http://hortsci.ashspublications.org/content/42/ 6/1317.full

Qin, H. N., and Shirley, G. (2007). Lagerstroemia Linnaeus. Flora China 13, 277-281. Available online at: http://foc.eflora.cn/cncontent.aspx?TaxonId= 117489

Raman, G., and Park, S. (2015). Analysis of the complete chloroplast genome of a medicinal plant, Dianthus superbus var. longicalyncinus, from a comparative genomics perspective. PLOS ONE 10:e0141329. doi: 10.1371/journal.pone.0141329

Raman, G., and Park, S. (2016). The complete chloroplast genome sequence of Ampelopsis: gene organization, comparative analysis, and phylogenetic relationships to other angiosperms. Front. Plant Sci. 7:341. doi: $10.3389 /$ fpls.2016.00341

Rambaut, A. (1996). Se-Al: Sequence Alignment Editor. Version 2.0. Oxford: University of Oxford, Department of Zoology.

Ronquist, F., Teslenko, M., van der Mark, P., Ayres, D. L., Darling, A., Hohna, S., et al. (2012). MrBayes 3.2: efficient Bayesian phylogenetic inference and model choice across a large model space. Syst. Biol. 61, 539-542. doi: 10.1093/sysbio/sys029

Song, Y., Dong, W., Liu, B., Xu, C., Yao, X., Gao, J., et al. (2015). Comparative analysis of complete chloroplast genome sequences of two tropical trees Machilus yunnanensis and Machilus balansae in the family Lauraceae. Front. Plant Sci. 6:662. doi: 10.3389/fpls.2015.00662

Stamatakis, A. (2006). RAxML-VI-HPC: maximum likelihood-based phylogenetic analyses with thousands of taxa and mixed models. Bioinformatics 22, 2688-2690. doi: 10.1093/bioinformatics/btl446

Su, H. J., Hogenhout, S. A., Al-Sadi, A. M., and Kuo, C. H. (2014). Complete chloroplast genome sequence of omani lime (Citrus aurantiifolia) and comparative analysis within the Rosids. PLoS ONE 9:e113049. doi: 10.1371/journal.pone.0113049

Suo, Z. L., Chen, L. N., Pei, D., Jin, X. B., and Zhang, H. J. (2015). A new nuclear DNA marker from ubiquitin ligase gene region for genetic diversity detection of walnut germplasm resources. Biotechnol. Rep. 5, 40-45. doi: $10.1016 /$ j.btre.2014.11.003

Suo, Z. L., Li, W. Y., Jin, X. B., and Zhang, H. J. (2016). A new nuclear DNA marker revealing both microsatellite variations and single nucleotide polymorphic loci: a case study on classification of cultivars in Lagerstroemia indica L. J. Microb. Biochem. Technol. 8, 266-271. doi: 10.4172/1948-5948.1000296

Suo, Z. L., Zhang, C. H., Zheng, Y. Q., He, L. X., Jin, X. B., Hou, B. X., et al. (2012). Revealing genetic diversity of tree peonies at micro-evolution level with hypervariable chloroplast markers and floral traits. Plant Cell Rep. 31, 2199-2213. doi: 10.1007/s00299-012-1330-0

Swofford, D. L. (2003). PAUP*. Phylogenetic Analysis Using Parsimony (* and Other Methods). Version4b10. Sunderland, MA: Sinauer.

Tamura, K., Stecher, G., Peterson, D., Filipski, A., and Kumar, S. (2013). MEGA6: molecular evolutionary genetics analysis version 6.0. Mol. Biol. Evol. 30, 2725-2729. doi: 10.1093/molbev/mst197

Thiel, T., Michalek, W., Varshney, R., and Graner, A. (2003). Exploiting EST databases for the development and characterization of gene-derived SSRmarkers in barley (Hordeum vulgare L.). Theor. Appl. Genet. 106, 411-422. doi: 10.1007/s00122-002-1031-0

Wang, X., Wadlb, P. A., Pounders, C., Trigiano, R. N., Cabrera, R. I., Scheffler, B. E., et al. (2011). Evaluation of genetic diversity and pedigree within crapemyrtle cultivars using simple sequence repeat markers. J. Amer. Soc. Hort. Sci. 136, 116-128. Available online at: http://journal.ashspublications.org/content/136/ 2/116.full

Wyman, S. K., Jansen, R. K., and Boore, J. L. (2004). Automatic annotation of organellar genomes with DOGMA. Bioinformatics 20, 3252-3255. doi: 10.1093/bioinformatics/bth352 
Xiang, X. G., Zhang, J. B., Lu, A. M., and Li, R. Q. (2011). Molecular identification on species in Juglandaceae: a tiered method. J. Syst. Evol. 49, 252-260. doi: 10.1111/j.1759-6831.2011. 00116.x

Yang, Y., Zhou, T., Duan, D., Yang, J., Feng, L., and Zhao, G. (2016). Comparative analysis of the complete chloroplast genomes of five Quercus species. Front. Plant Sci. 7:959. doi: 10.3389/fpls.2016. 00959

Yang, Z. H. (2007). PAML 4: Phylogenetic analysis by maximum likelihood. Mol. Biol. Evol. 24, 1586-1591. doi: 10.1093/molbev/ msm088

Zhang, Y., Du, L., Liu, A., Chen, J., Wu, L., Hu, W., et al. (2016). The complete chloroplast genome sequences of five Epimedium species: lights into phylogenetic and taxonomic analyses. Front. Plant Sci. 7:306 doi: $10.3389 /$ fpls.2016.00306

Conflict of Interest Statement: The authors declare that the research was conducted in the absence of any commercial or financial relationships that could be construed as a potential conflict of interest.

Copyright (C) 2017 Xu, Dong, Li, Lu, Xie, Jin, Shi, He and Suo. This is an open-access article distributed under the terms of the Creative Commons Attribution License (CC $B Y)$. The use, distribution or reproduction in other forums is permitted, provided the original author(s) or licensor are credited and that the original publication in this journal is cited, in accordance with accepted academic practice. No use, distribution or reproduction is permitted which does not comply with these terms. 